PROCEEDINGS OF THE

AMERICAN MATHEMATICAL SOCIETY

Volume 131, Number 12, Pages 3901-3912

S 0002-9939(03)06951-X

Article electronically published on February 24, 2003

\title{
TRIVIAL, STRONGLY MINIMAL THEORIES ARE MODEL COMPLETE AFTER NAMING CONSTANTS
}

\author{
SERGEY S. GONCHAROV, VALENTINA S. HARIZANOV, MICHAEL C. LASKOWSKI, \\ STEFFEN LEMPP, AND CHARLES F. D. MCCOY \\ (Communicated by Carl G. Jockusch, Jr.)
}

\begin{abstract}
We prove that if $\mathcal{M}$ is any model of a trivial, strongly minimal theory, then the elementary diagram $\operatorname{Th}\left(\mathcal{M}_{M}\right)$ is a model complete $\mathcal{L}_{M}$-theory. We conclude that all countable models of a trivial, strongly minimal theory with at least one computable model are $\mathbf{0}^{\prime \prime}$-decidable, and that the spectrum of computable models of any trivial, strongly minimal theory is $\Sigma_{5}^{0}$.
\end{abstract}

The purely model-theoretic result in the title was not arrived at in a straightforward way. Rather, it arose from a question in computable model theory raised by the fourth author of this paper. In discussions with various colleagues over several years, he had been unable to "code complicated sets into" countable models of uncountably categorical theories. In the end, as our result shows, it turned out that there was a purely model-theoretic reason for his failure.

Since this paper is intended for two distinct audiences, we organize the remainder of it as follows. Section 1 recalls some model-theoretic definitions, states and proves the Main Theorem, and derives some model-theoretic consequences that are used in the next section. Then Section 2 presents the motivation from, and some consequences in, computable model theory.

For basic definitions and results, we refer the reader to Chang/Keisler [3], Buechler [2], and Pillay [17] in model theory, and Ershov/Goncharov [4] and Harizanov [8] in computable model theory.

\section{The Main Theorem And its Proof}

Throughout the paper, we assume a countable first-order language $\mathcal{L}$. We recall some basic model-theoretic notions for the convenience of the reader.

Received by the editors February 28, 2002 and, in revised form, June 25, 2002.

2000 Mathematics Subject Classification. Primary 03C10; Secondary 03C35, 03 C57.

Key words and phrases. Strongly minimal, trivial geometry, uncountably categorical, model complete, computable model, spectrum of computable models.

This research was partially supported by the NSF Binational Grant DMS-0075899.

The first author's research was also partially supported by the Russian Foundation for Basic Research grant 99-01-00485. The third author's research was partially supported by NSF grant DMS-0071746. The fourth author's research was partially supported by NSF grant DMS-9732526 and by the Vilas Foundation of the University of Wisconsin. The fifth author's research was partially supported by an NSF VIGRE Fellowship.

The fourth author would also like to thank numerous other model theorists with whom he had discussed this problem over the past few years. 
Definition 1. (1) A complete theory $T$ is strongly minimal if any definable subset of any model $\mathcal{M}$ of $T$ is finite or cofinite. (Here and throughout, "definable" means "definable with parameters".) We call a structure $\mathcal{M}$ strongly minimal if it has a strongly minimal theory.

(2) A strongly minimal model $\mathcal{M}$ is trivial (or, more precisely, has trivial pregeometry) if for all subsets $A \subseteq M$,

$$
\operatorname{acl}(A)=\bigcup_{a \in A} \operatorname{acl}(\{a\}) .
$$

Note that a strongly minimal theory in a countable language is uncountably categorical, and that triviality is really a property of the theory of a model rather than of the model itself.

Roughly speaking, strongly minimal theories can be classified into three kinds: trivial, locally modular nontrivial, and non-locally modular. Canonical examples of these three kinds of theories are the theory of $\langle\omega, S\rangle$ ( $\omega$ with successor function), the theory of a vector space over a fixed field, and the theory of algebraically closed fields of a fixed characteristic, respectively.

We will frequently use the concept of expansion by constants. We set notation in the following.

Definition 2. Given a model $\mathcal{M}$ and a subset $X \subseteq M$, the expansion $\mathcal{M}_{X}$ of $\mathcal{M}$ by constants in $X$ is obtained by adding constant symbols for each $x \in X$ (interpreted in the canonical way). We denote the corresponding expansion of the first-order language $\mathcal{L}$ by $\mathcal{L}_{X}$. The elementary diagram of $\mathcal{M}$ is the $\mathcal{L}_{M}$-theory $\operatorname{Th}\left(\mathcal{M}_{M}\right)$.

Note that expansion by constants preserves strong minimality and triviality.

We can now state our

Main Theorem. For any trivial, strongly minimal theory $T$, the elementary diagram of any model $\mathcal{M}$ of $T$ is a model complete $\mathcal{L}_{M}$-theory.

Note that a trivial, strongly minimal model need not be model complete in its original language, e.g., $\langle\omega, S\rangle$ ( $\omega$ with successor function) is not model complete. We also note that the triviality of $T$ is used only once in our proof, namely, in Case 2 of the proof of Lemma 11. Finally, strong minimality is necessary in the hypothesis of the Main Theorem by Marker [15], who showed that for every $n \in \omega$, there is an almost strongly minimal $\aleph_{0}$-categorical (and thus also $\aleph_{1}$-categorical) theory which is not $\Sigma_{n}$-axiomatizable, while we show this to be false for trivial, strongly minimal theories in Corollary 18

For the proof of our theorem, fix any model $\mathcal{M}_{0}$ of a trivial, strongly minimal theory $T$. We will argue that the elementary diagram of $\mathcal{M}_{0}$ is model complete.

To simplify the notation, let $T^{*}$ denote the theory $\operatorname{Th}\left(\left(\mathcal{M}_{0}\right)_{M_{0}}\right)$ and let $\mathcal{L}^{*}$ be the language of $T^{*}$ (i.e., $\mathcal{L}^{*}=\mathcal{L}_{M_{0}}$ ). In everything that follows, we will be working with the theory $T^{*}$ in the language $\mathcal{L}^{*}$.

Additionally, fix a cardinal $\kappa>\left|M_{0}\right|$ and fix models $\mathcal{M} \subseteq \mathcal{N}$ of $T^{*}$, each of size $\kappa$. As both $\mathcal{M}$ and $\mathcal{N}$ are models of $T^{*}$, we may assume that $\mathcal{M}_{0} \preceq \mathcal{M}$ and $\mathcal{M}_{0} \preceq \mathcal{N}$. The entire argument is devoted to showing that $\mathcal{M} \preceq \mathcal{N}$. It is well known (see, e.g., Chang/Keisler [3, Corollary 3.5.3]) that the model completeness of $T^{*}$ follows from this. 
In what follows, we require two standard facts about strongly minimal theories. The first is known as the non-finite cover property, which holds for any uncountably categorical theory (see, e.g., Shelah [18, Lemma IX.1.10]). In our context, it reads as follows.

Lemma 3. For all $\mathcal{L}^{*}$-formulas $\varphi(\bar{x}, \bar{y})$, there is a number $k$ such that for every $\mathcal{M}^{*}=T^{*}$ and every $\bar{b}$ from $M^{*}$, either $\varphi\left(\bar{b}, \mathcal{M}^{*}\right)$ is infinite or has size at most $k$. Furthermore, the number $k$ depends only on $\varphi$ and the partition of the free variables into $(\bar{x}, \bar{y})$.

Thus, we are permitted to use the quantifiers $\exists^{<\infty}$ and $\exists^{\infty}$, where $\exists^{<\infty} \bar{y} \varphi(\bar{x}, \bar{y})$ is shorthand for $\exists \leq k \bar{y} \varphi(\bar{x}, \bar{y})$, and $\exists^{\infty} \bar{y} \varphi(\bar{x}, \bar{y})$ is shorthand for $\neg^{<\infty} \bar{y} \varphi(\bar{x}, \bar{y})$. We note a very Simple Observation, which is just an application of the pigeon-hole principle. However, we distinguish it as a lemma so that we can refer to it later.

Lemma 4. If $\mathcal{N} \models \exists^{\infty} \bar{y} \varphi(\bar{b}, \bar{y})$ with $\lg (\bar{y})=k+1$, then there is a partition of $\bar{y}$ into $w \bar{z}$ with $\lg (w)=1$ and $\lg (\bar{z})=k$ such that $\mathcal{N} \models \exists^{\infty} w \exists \bar{z} \varphi(\bar{b}, w, \bar{z})$. (The variable $w$ need not be the first element of $\bar{y}$, but it simplifies notation to write it as if it were.)

The second basic fact goes by the name of "Finite Satisfiability". The general formulation is that if $\mathcal{M}_{0} \preceq \mathcal{N}$ are models of a stable theory and $\mathcal{N} \models \varphi(\bar{b}, \bar{c})$ for some $\mathcal{L}_{M_{0}}$-formula and some $\bar{b}, \bar{c}$ from $N$ that are independent (i.e., do not fork over $\left.M_{0}\right)$, then there is $\bar{a}$ from $M_{0}$ such that $\mathcal{N} \models \varphi(\bar{a}, \bar{c})$. This fact follows easily from the fact that in a stable theory, every complete type over a model is definable (see, e.g., Pillay [17, Corollary 1.21]). As this is an important point, we record how this is manifested in the context of strongly minimal theories with the lemma below.

Lemma 5. Suppose that $\mathcal{M}_{0} \preceq \mathcal{N}$ are models of a strongly minimal theory, $\bar{b}$ and $\bar{c}$ are tuples from $N$ such that at least one of $\bar{b}, \bar{c} \subseteq \operatorname{acl}\left(M_{0} e\right)$ for a single element $e$, and $\operatorname{acl}\left(M_{0} \bar{b}\right) \cap \operatorname{acl}\left(M_{0} \bar{c}\right)=M_{0}$. If $\mathcal{N} \models \varphi(\bar{b}, \bar{c})$ for an $\mathcal{L}_{M_{0}}$-formula $\varphi(\bar{x}, \bar{y})$, then there is $\bar{a}$ from $M_{0}$ such that $\mathcal{N} \models \varphi(\bar{a}, \bar{c})$.

Proof. By symmetry, suppose that $\bar{b} \subseteq \operatorname{acl}\left(M_{0} b\right)$, where $b$ is a single element. From the note above, it suffices to show that $\bar{b}$ is independent from $\bar{c}$ over $M_{0}$. But, if $\bar{b}$ were not independent from $\bar{c}$ over $M_{0}$, then (since $\bar{b} \subseteq \operatorname{acl}\left(M_{0} b\right)$ ) $b$ would not be independent from $\bar{c}$ over $M_{0}$. Since $b$ is a single element and the theory is strongly minimal, this would imply $b \in \operatorname{acl}\left(M_{0} \bar{c}\right)$, so $\bar{b} \subseteq \operatorname{acl}\left(M_{0} \bar{c}\right)$.

The following well-known notion is crucial for the rest of this section.

Definition 6. An $\mathcal{L}^{*}$-formula $\varphi(\bar{x})$ is absolute if for all $\bar{b}$ from $M, \mathcal{M} \models \varphi(\bar{b})$ if and only if $\mathcal{N}=\varphi(\bar{b})$.

To complete our proof that $\mathcal{M} \preceq \mathcal{N}$, it suffices to show that every $\mathcal{L}^{*}$-formula is absolute. Clearly, every quantifier-free $\mathcal{L}^{*}$-formula is absolute. Similarly, the family of absolute formulas is closed under the Boolean operations. Thus, to complete our proof that $T^{*}$ is model complete, it suffices to show that if an $\mathcal{L}^{*}$-formula $\varphi(\bar{x}, y)$ is absolute, then $\exists y \varphi(\bar{x}, y)$ is absolute as well. The bulk of this section provides a verification of this claim. 
Definition 7. An $\mathcal{L}^{*}$-formula $\varphi(\bar{x}, \bar{y})$ is an $(n, m)$-formula if $\lg (\bar{x})=n$ and $\lg (\bar{y})=$ $m$. We identify three interrelated families of statements:

- $A_{n, m}$, the statement that for all absolute $(n, m)$-formulas $\varphi(\bar{x}, \bar{y})$, the formula $\exists<\infty \bar{y} \varphi(\bar{x}, \bar{y})$ is absolute.

- $B_{n, m}$, the statement that for all absolute $(n, m)$-formulas $\varphi(\bar{x}, \bar{y})$, if $\bar{b} \in M^{n}$ and $\mathcal{N} \models \exists^{<\infty} \bar{y} \varphi(\bar{b}, \bar{y})$, then $\varphi(\bar{b}, \mathcal{N})=\varphi(\bar{b}, \mathcal{M})$, i.e., every realization of $\varphi(\bar{b}, \bar{y})$ in $N^{m}$ is an element of $M^{m}$.

- $C_{n, m}$, the statement that for all absolute $(n, m)$-formulas $\varphi(\bar{x}, \bar{y})$, the formula $\exists \bar{y} \varphi(\bar{x}, \bar{y})$ is absolute.

In light of our observations above, showing that $T^{*}$ is model complete amounts to showing that $C_{n, 1}$ holds for all $n \in \omega$. Clearly, by simply adding dummy variables, each of the three classes of properties is preserved by decreasing the indices (e.g., $B_{n, m}$ implies $B_{n^{\prime}, m^{\prime}}$ for all $n^{\prime} \leq n$ and all $m^{\prime} \leq m$ ).

Lemma 8. For all $n, m \in \omega, B_{n, m}$ implies $C_{n, m}$.

Proof. Fix $n$ and $m$ and assume that $B_{n, m}$ holds. We will prove by induction that $C_{n, k}$ holds for all $k \leq m$. To begin, note that $C_{n, 0}$ is vacuously true (since $\exists \bar{y} \varphi(\bar{x}, \bar{y})$ is simply $\varphi(\bar{x})$ when $\lg (\bar{y})=0)$. So assume that $B_{n, m}$ and $C_{n, k}$ hold, where $k<m$. We argue that $C_{n, k+1}$ holds. Fix an absolute $(n, k+1)$-formula $\varphi(\bar{x}, \bar{y})$ and $\bar{b} \in M^{n}$ such that $\mathcal{N}=\exists \bar{y} \varphi(\bar{b}, \bar{y})$. Our proof splits into two cases.

Case 1. $\mathcal{N} \models \exists^{<\infty} \bar{y} \varphi(\bar{b}, \bar{y})$.

Then, since $B_{n, k+1}$ holds (recall $k+1 \leq m$ ), we have $\varphi(\bar{b}, \mathcal{N})=\varphi(\bar{b}, \mathcal{M})$, hence there is $\bar{c} \in M^{k+1}$ such that $\mathcal{M}=\varphi(\bar{b}, \bar{c})$. Thus, $\mathcal{M}=\exists \bar{y} \varphi(\bar{b}, \bar{y})$.

Case 2. $\mathcal{N} \models \exists^{\infty} \bar{y} \varphi(\bar{b}, \bar{y})$.

By the Simple Observation (Lemma 44), there is a partition of $\bar{y}$ into $w \bar{z}$ with $\lg (w)=1$ and $\lg (\bar{z})=k$ such that $\mathcal{N} \models \exists^{\infty} w \exists \bar{z} \varphi(\bar{b}, w, \bar{z})$. Thus, by strong minimality, $\{e \in N \mid \mathcal{N} \models \exists \bar{z} \varphi(\bar{b}, e, \bar{z})\}$ is cofinite, so there is $a \in M_{0}$ such that $\mathcal{N} \models \exists \bar{z} \varphi(\bar{b}, a, \bar{z})$. Let $\psi(\bar{x}, \bar{z})$ be the $(n, k)$-formula $\varphi(\bar{x}, a, \bar{z})$. Since $\varphi$ is absolute, $\psi$ is absolute, so, by $C_{n, k}, \exists \bar{z} \psi$ is absolute. Thus, there is $\bar{c} \in M^{k}$ such that $\mathcal{M} \models \varphi(\bar{b}, a, \bar{c})$. So $a \bar{c}$ witnesses $\mathcal{M} \models \exists \bar{y} \varphi(\bar{b}, \bar{y})$.

Lemma 9. For all $n, m \in \omega, B_{n, m}$ implies $A_{n, m+1}$.

Proof. Fix $n$ and $m$ such that $B_{n, m}$ holds. Note that $C_{n, m}$ holds by Lemma 8 Fix an absolute $(n, m+1)$-formula $\varphi(\bar{x}, \bar{y})$ and choose $\bar{b} \in M^{n}$. Clearly, if $\mathcal{N} \models$ $\exists^{<\infty} \bar{y} \varphi(\bar{b}, \bar{y})$, then $\mathcal{M}=\exists^{<\infty} \bar{y} \varphi(\bar{b}, \bar{y})$. So assume $\mathcal{N}=\exists^{\infty} \bar{y} \varphi(\bar{b}, \bar{y})$. It follows from the Simple Observation that there is a partition $\bar{y}$ into $w \bar{z}$ with $\lg (w)=1$ and $\lg (\bar{z})=m$ such that $\mathcal{N} \models \exists^{\infty} w \exists \bar{z} \varphi(\bar{b}, w, \bar{z})$. Thus, $\{e \in N \mid \mathcal{N} \models \exists \bar{z} \varphi(\bar{b}, e, \bar{z})\}$ is cofinite, so there is an infinite subset $\left\{a_{i} \mid i \in \omega\right\}$ of $M_{0}$ such that $\mathcal{N} \models \exists \bar{z} \varphi\left(\bar{b}, a_{i}, \bar{z}\right)$ for each $i$. For $i \in \omega$, let $\psi_{i}(\bar{x}, \bar{z})$ be the $(n, m)$-formula $\varphi\left(\bar{x}, a_{i}, \bar{z}\right)$. Since $\varphi$ is absolute, each $\psi_{i}$ is absolute, so, by $C_{n, m}, \exists \bar{z} \psi_{i}$ is absolute for each $i$. Thus, for each $i$, there is $\bar{c}_{i} \in M^{m}$ such that $\mathcal{M} \models \varphi\left(\bar{b}, a_{i}, \bar{c}_{i}\right)$. The sequence $\left\langle a_{i} \bar{c}_{i} \mid i \in \omega\right\rangle$ witnesses $\mathcal{M}=\exists^{\infty} \bar{y} \varphi(\bar{b}, \bar{y})$.

Lemma 10. For all $m \in \omega, B_{1, m}$ (and hence $B_{0, m}$ ) holds.

Proof. Let $\varphi(x, \bar{y})$ be an absolute $(1, m)$-formula and choose $b \in M$ such that $\mathcal{N} \models \exists^{<\infty} \bar{y} \varphi(b, \bar{y})$. Choose $r \in \omega$ such that $\mathcal{N} \models \exists^{=r} \bar{y} \varphi(b, \bar{y})$. We argue that $\mathcal{M} \models \exists^{=r} \bar{y} \varphi(b, \bar{y})$ by splitting into two cases. On the one hand, if $b \in M_{0}$, then 
$\exists=r \bar{y} \varphi(b, \bar{y})$ is an $\mathcal{L}^{*}$-sentence true in $\mathcal{N}$, so $\exists^{=r} \bar{y} \varphi(b, \bar{y}) \in T^{*}$, hence is satisfied by $\mathcal{M}$. On the other hand, if $b \notin M_{0}$, then, by strong minimality, there is a finite subset $\left\{a_{j} \mid j<s\right\}$ of $M_{0}$ such that

$$
T^{*} \models \forall x\left(\bigwedge_{j<s} x \neq a_{j} \rightarrow \exists^{=r} \bar{y} \varphi(x, \bar{y})\right),
$$

so $\mathcal{M} \models \exists^{=r} \bar{y} \varphi(b, \bar{y})$ as desired.

Now choose distinct $\bar{c}_{0}, \ldots, \bar{c}_{r-1} \in M^{m}$ such that $\mathcal{M}=\varphi\left(b, \bar{c}_{i}\right)$ for each $i<r$. By absoluteness, $\mathcal{N} \models \varphi\left(b, \bar{c}_{i}\right)$ for each $i<r$, so $\varphi(b, \mathcal{N})=\left\{\bar{c}_{i} \mid i<r\right\}=\varphi(b, \mathcal{M})$.

Proposition 11. For all $n, m \in \omega, B_{n, m+1}$ and $A_{n+1, m}$ imply $B_{n+1, m}$.

Proof. In light of Lemma 10, we may assume $n \geq 1$. Choose an absolute $\mathcal{L}^{*}$-formula $\varphi(\bar{x}, y, \bar{z})$, where $\lg (\bar{x})=n, \lg (y)=1$, and $\lg (\bar{z})=m$. Choose any $\bar{b} \in M^{n}$ and $b_{2} \in M$ such that $\mathcal{N} \models \exists<\infty \bar{z} \varphi\left(\bar{b}, b_{2}, \bar{z}\right)$, and fix a witness $\bar{c}^{*} \in N^{m}$ such that $\mathcal{N} \models \varphi\left(\bar{b}, b_{2}, \bar{c}^{*}\right)$. We will eventually show that $\bar{c}^{*} \in M^{m}$.

To start, fix any element $e^{*} \in M \backslash \operatorname{acl}\left(M_{0} \bar{b}\right)$. (For the whole of this proposition, we compute algebraic closures in the model $\mathcal{N}$.) Such an element exists because $\left|\operatorname{acl}\left(M_{0} \bar{b}\right)\right|=\left|\operatorname{acl}\left(M_{0}\right)\right|<\kappa=|M|$. Our argument splits into two cases.

Case 1. $\mathcal{N} \models \exists^{\infty} \bar{z} \varphi\left(\bar{b}, e^{*}, \bar{z}\right)$.

It follows, by strong minimality and since $e^{*} \notin \operatorname{acl}\left(M_{0} \bar{b}\right)$, that the set of all elements $d \in N$ with $\mathcal{N} \models \exists<\infty \bar{z} \varphi(\bar{b}, d, \bar{z})$ is finite. Let $\psi(\bar{x}, y, \bar{z})$ denote the $\mathcal{L}^{*}$ formula

$$
\varphi(\bar{x}, y, \bar{z}) \wedge \exists^{<\infty} \bar{w} \varphi(\bar{x}, y, \bar{w}) .
$$

Clearly, $\mathcal{N} \models \psi\left(\bar{b}, b_{2}, \bar{c}^{*}\right)$. Since $A_{n+1, m}$ holds (and since absolute formulas are closed under Boolean operations), $\psi$ is absolute. Let

$$
D=\{d \in N|\mathcal{N}|=\exists \bar{z} \psi(\bar{b}, d, \bar{z})\} .
$$

It follows from the first observation of this case that $D$ is finite. Thus, the set

$$
E=\left\{d \bar{e} \in N^{m+1} \mid \mathcal{N} \models \psi(\bar{b}, d, \bar{e})\right\}
$$

is finite as well. That is, $\mathcal{N} \models \exists^{<\infty} y \bar{z} \psi(\bar{b}, y, \bar{z})$. But $B_{n, m+1}$ holds, hence every solution to this formula is in $M$. In particular, $b_{2} \bar{c}^{*} \in M^{m+1}$, so $\bar{c}^{*} \in M^{m}$.

Case 2. $\mathcal{N}=\exists^{<\infty} \bar{z} \varphi\left(\bar{b}, e^{*}, \bar{z}\right)$.

Let $\left\langle\bar{c}_{j} \mid j<r\right\rangle$ be the realizations of $\varphi\left(\bar{b}, e^{*}, \bar{z}\right)$ in $N^{m}$. Now fix $j<r$. Write $\bar{c}_{j}$ as $\left\langle c_{i, j} \mid i<m\right\rangle$. Let $\bar{d}_{j}$ denote the subsequence of $\bar{c}_{j}$ containing the $c_{i, j}$ 's that satisfy $c_{i, j} \in \operatorname{acl}\left(M_{0} \bar{b}\right)$ and $\bar{u}_{j}$ denote the corresponding subsequence of $\bar{z}$. That is, $\bar{u}_{j}$ consists of the $z_{i}$ for which $c_{i, j} \in \operatorname{acl}\left(M_{0} \bar{b}\right)$. Dually, let $\bar{e}_{j}$ be the 'complement' of $\bar{d}_{j}$. That is, $\bar{e}_{j}$ is the subsequence of $\bar{c}_{j}$ containing all of the $c_{i, j} \notin \operatorname{acl}\left(M_{0} \bar{b}\right)$. Let $\bar{v}_{j}$ denote the corresponding subsequence of $\bar{z}$. For notational convenience, we write $\bar{c}_{j}=\bar{d}_{j} \bar{e}_{j}$ and $\bar{z}=\bar{u}_{j} \bar{v}_{j}$, although there is no reason why $\bar{d}_{j}$ should be an initial segment of $\bar{c}_{j}$.

By triviality, every element of $\bar{e}_{j}$ is in $\operatorname{acl}\left(M_{0} e^{*}\right)$. (To see this, the formula $\varphi\left(\bar{b}, e^{*}, \bar{z}\right)$ demonstrates that each element of $\bar{e}_{j}$ is in $\operatorname{acl}\left(M_{0} \bar{b} e^{*}\right)$. But triviality implies that $\operatorname{acl}\left(M_{0} \bar{b} e^{*}\right)=\operatorname{acl}\left(M_{0} \bar{b}\right) \cup \operatorname{acl}\left(M_{0} e^{*}\right)$ and the elements of $\bar{e}_{j}$ were chosen to be outside of $\operatorname{acl}\left(M_{0} \bar{b}\right)$.) In particular, it follows that

$$
\operatorname{acl}\left(M_{0} \overline{b d}_{j}\right) \cap \operatorname{acl}\left(M_{0} e^{*} \bar{e}_{j}\right)=M_{0} .
$$


(To see this, suppose we have $f \in \operatorname{acl}\left(M_{0} \overline{b d}_{j}\right) \cap \operatorname{acl}\left(M_{0} e^{*} \bar{e}_{j}\right)$. Then $f \in \operatorname{acl}\left(M_{0} \bar{b}\right) \cap$ $\operatorname{acl}\left(M_{0} e^{*}\right)$. So if $f \notin M_{0}$ then, by exchange,

$$
e^{*} \in \operatorname{acl}\left(M_{0} f\right) \subseteq \operatorname{acl}\left(M_{0} \bar{b}\right),
$$

which is contrary to our choice of $e^{*}$.)

Clearly,

$$
\mathcal{N} \models \varphi\left(\bar{b}, e^{*}, \bar{d}_{j}, \bar{e}_{j}\right) \wedge \exists^{<\infty} \bar{u}_{j} \varphi\left(\bar{b}, e^{*}, \bar{u}_{j}, \bar{e}_{j}\right) .
$$

So, by finite satisfiability (Lemma 5), there is $\left(\hat{a}_{j}, \bar{a}_{j}\right)$ (corresponding to $\left(e^{*}, \bar{e}_{j}\right)$ ) from $M_{0}$ such that

$$
\mathcal{N}=\varphi\left(\bar{b}, \hat{a}_{j}, \bar{d}_{j}, \bar{a}_{j}\right) \wedge \exists^{<\infty} \bar{u}_{j} \varphi\left(\bar{b}, \hat{a}_{j}, \bar{u}_{j}, \bar{a}_{j}\right) .
$$

Let $\psi_{j}\left(\bar{x}, \bar{u}_{j}\right)$ denote the $\mathcal{L}^{*}$-formula $\varphi\left(\bar{x}, \hat{a}_{j}, \bar{u}_{j}, \bar{a}_{j}\right)$.

Similarly, by finite satisfiability again, there is $\left(\bar{a}_{j}^{1}, \bar{a}_{j}^{2}\right)$ from $M_{0}$ (corresponding to $\left.\left(\bar{b}, \bar{d}_{j}\right)\right)$ such that

$$
\mathcal{N} \models \varphi\left(\bar{a}_{j}^{1}, e^{*}, \bar{a}_{j}^{2}, \bar{e}_{j}\right) \wedge \exists^{<\infty} \bar{v}_{j} \varphi\left(\bar{a}_{j}^{1}, e^{*}, \bar{a}_{j}^{2}, \bar{v}_{j}\right) .
$$

Let $\theta_{j}\left(y, \bar{v}_{j}\right)$ denote the $\mathcal{L}^{*}$-formula $\varphi\left(\bar{a}_{j}^{1}, y, \bar{a}_{j}^{2}, \bar{v}_{j}\right)$ and let

$$
\delta_{j}(\bar{x}, y, \bar{z}):=\psi_{j}\left(\bar{x}, \bar{u}_{j}\right) \wedge \exists^{<\infty} \bar{u}_{j} \psi_{j}\left(\bar{x}, \bar{u}_{j}\right) \wedge \theta_{j}\left(y, \bar{v}_{j}\right) \wedge \exists^{<\infty} \bar{v}_{j} \theta_{j}\left(y, \bar{v}_{j}\right) .
$$

Note that

$$
\mathcal{N} \models \psi_{j}\left(\bar{b}, \bar{d}_{j}\right) \wedge \exists^{<\infty} \bar{u}_{j} \psi_{j}\left(\bar{b}, \bar{u}_{j}\right) \wedge \theta_{j}\left(e^{*}, \bar{e}_{j}\right) \wedge \exists^{<\infty} \bar{v}_{j} \theta_{j}\left(e^{*}, \bar{v}_{j}\right),
$$

hence $\mathcal{N} \models \delta_{j}\left(\bar{b}, e^{*}, \bar{c}_{j}\right)$. Since $A_{n, m}$ holds, the $\mathcal{L}^{*}$-formulas $\psi_{j}, \theta_{j}$, and $\delta_{j}$ are all absolute.

Now, returning to our original scenario, suppose $\mathcal{N} \models \delta_{j}\left(\bar{b}, b_{2}, \bar{c}^{*}\right)$ for some $j<r$. Let $\bar{d}^{*}$ denote the subsequence of $\bar{c}^{*}$ (corresponding to $\bar{u}_{j}$ ) and let $\bar{e}^{*}$ denote the subsequence of $\bar{c}^{*}$ (corresponding to $\bar{v}_{j}$ ). Then

$$
\mathcal{N} \models \psi_{j}\left(\bar{b}, \bar{d}^{*}\right) \wedge \exists^{<\infty} \bar{u}_{j} \psi_{j}\left(\bar{b}, \bar{u}_{j}\right) .
$$

Thus, since $B_{n, m}$ holds, $\bar{d}^{*} \in M^{\lg \left(\bar{u}_{j}\right)}$. Similarly,

$$
\mathcal{N} \models \theta_{j}\left(b_{2}, \bar{e}^{j}\right) \wedge \exists^{<\infty} \bar{v}_{j} \theta_{j}\left(b_{2}, \bar{v}_{j}\right),
$$

so, since $B_{1, m}$ holds, $\bar{e}^{*} \in M^{\lg \left(\bar{v}_{j}\right)}$. Hence $\bar{c}^{*} \in M^{m}$ as required.

Finally, suppose that $\mathcal{N} \models \neg \delta_{j}\left(\bar{b}, b_{2}, \bar{c}^{*}\right)$ for all $j<r$. Then $\mathcal{N} \models \eta\left(\bar{b}, b_{2}, \bar{c}^{*}\right)$, where

$$
\eta(\bar{x}, y, \bar{z}):=\varphi(\bar{x}, y, \bar{z}) \wedge \exists^{<\infty} \bar{z} \varphi(\bar{x}, y, \bar{z}) \wedge \bigwedge_{j<r} \neg \delta_{j}\left(\bar{x}, y, \bar{z}_{j}\right) .
$$

It follows from $A_{n+1, m}$ and the absoluteness of $\varphi$ and the $\delta_{j}$ 's that $\eta$ is absolute.

We claim that $\mathcal{N} \models \exists^{<\infty} y \bar{z} \eta(\bar{b}, y, \bar{z})$. To see this, let

$$
F=\{f \in N \mid \mathcal{N} \models \exists \bar{z} \eta(\bar{b}, f, \bar{z})\} .
$$

If $F$ were infinite, then $\mathcal{N} \models \exists \bar{z} \eta(\bar{b}, f, \bar{z})$ would hold for every $f \in N \backslash \operatorname{acl}\left(M_{0} \bar{b}\right)$. In particular, $\mathcal{N} \models \exists \bar{z} \eta\left(\bar{b}, e^{*}, \bar{z}\right)$. But what could such a solution be? Since $\varphi(\bar{x}, y, \bar{z})$ is a conjunct of $\eta$, the only possible solutions to $\eta\left(\bar{b}, e^{*}, \bar{z}\right)$ could be $\left\{\bar{c}_{j} \mid j<r\right\}$. But $\mathcal{N} \models \delta_{j}\left(\bar{b}, e^{*}, \bar{c}_{j}\right)$ for each $j$, hence $\mathcal{N} \models \neg \exists \bar{z} \eta\left(\bar{b}, e^{*}, \bar{z}\right)$. Thus $F$ must be finite. However, for any $f \in F$ there are only finitely many $\bar{h} \in N^{m}$ such that $\mathcal{N} \models \eta(\bar{b}, f, \bar{h})$. Thus there are only finitely many tuples $f \bar{h} \in N^{m+1}$ that satisfy $\eta(\bar{b}, y, \bar{z})$ in $\mathcal{N}$. That is, $\mathcal{N} \models \exists<\infty y \bar{z} \eta(\bar{b}, y, \bar{z})$. 
Now, since $B_{n, m+1}$ holds, every solution to $\eta(\bar{b}, y, \bar{z})$ lies in $M$. In particular, $b_{2} \bar{c}^{*} \in M^{m+1}$, so $\bar{c}^{*} \in M^{m}$, completing the proof.

Proof of the Main Theorem. As noted earlier, the model completeness of $T^{*}$ follows from a demonstration of $\mathcal{M} \preceq \mathcal{N}$. We first show that $B_{n, m}$ must hold for all $n, m \in \omega$. To see this, we argue, by induction on $n$, that $B_{n, m}$ holds for all $m$. Lemma 10 provides the base case, i.e., $B_{1, m}$ holds for all $m \in \omega$. So fix $n \geq 1$ and assume that $B_{n, m}$ holds for all $m$. We prove that $B_{n+1, m}$ holds for all $m$, by induction on $m$. First, $B_{n+1,0}$ holds vacuously. So assume that $B_{n+1, m}$ holds for some $m$. Then $B_{n, m+2}$ holds by our inductive assumption and $A_{n+1, m+1}$ holds by Lemma 9 since $B_{n+1, m}$ holds. So $B_{n+1, m+1}$ holds by Proposition 11, and our induction is complete.

But now, Lemma 8 implies that $C_{n, m}$ holds for all $n, m \in \omega$. In particular, $C_{n, 1}$ holds for all $n \in \omega$. But this precisely says that the family of absolute $\mathcal{L}^{*}$ formulas is closed under existential quantification. As we already knew that the family of absolute $\mathcal{L}^{*}$-formulas contains the quantifier-free formulas and is closed under Boolean connectives, we conclude that every $\mathcal{L}^{*}$-formula is absolute. Thus, $\mathcal{M} \preceq \mathcal{N}$ as required.

We conclude this section with some observations about this variant of model completeness, which has been studied by Kueker in work that has not yet been published [14. All of the results in the remainder of this section are variants of theorems therein, but we include proofs for completeness.

Definition 12. For any structure $\mathcal{M}$, let $\operatorname{Th}_{\forall \exists}\left(\mathcal{M}_{M}\right)$ be the set of all $\forall \exists$-sentences $\sigma \in \operatorname{Th}\left(\mathcal{M}_{M}\right)$ (i.e., in the language $\left.\mathcal{L}_{M}\right)$.

Lemma 13. If the elementary diagram of a structure $\mathcal{M}$ is model complete, then $\operatorname{Th}\left(\mathcal{M}_{M}\right)$ is $\forall \exists$-axiomatizable.

Proof. Well-known (see, e.g., Chang/Keisler [3, Proposition 3.5.10]).

In addition to providing a criterion that will be useful in the next section, the following proposition demonstrates that the model completeness of the elementary diagram of a structure $\mathcal{M}$ is a property of the theory of $\mathcal{M}$. First, we need a definition.

Definition 14. An $\forall \exists$-formula $\theta(\bar{y})$ of $\mathcal{L}$ and an existential $\mathcal{L}$-formula $\psi(\bar{x}, \bar{y})$ form a linked pair $(\theta, \psi)$ (for $T)$ if

(1) $T \models \exists \bar{y} \theta(\bar{y})$ and

(2) $T \models \forall \bar{y} \forall \bar{y}^{\prime} \forall \bar{x}\left[\theta(\bar{y}) \wedge \theta\left(\bar{y}^{\prime}\right) \wedge \psi(\bar{x}, \bar{y}) \rightarrow \psi\left(\bar{x}, \bar{y}^{\prime}\right)\right]$.

Proposition 15. The elementary diagram of an $\mathcal{L}$-structure $\mathcal{M}$ is model complete if and only if, for every $\mathcal{L}$-formula $\varphi(\bar{x})$, there is a linked pair $(\theta, \psi)$ for the theory of $\mathcal{M}$ such that

$$
\mathcal{M}=\forall \bar{y}(\theta(\bar{y}) \rightarrow \forall \bar{x}[\varphi(\bar{x}) \leftrightarrow \psi(\bar{x}, \bar{y})]) .
$$

Proof. First, suppose that the elementary diagram of $\mathcal{M}$ is model complete. Fix an $\mathcal{L}$-formula $\varphi(\bar{x})$. Since $\operatorname{Th}\left(\mathcal{M}_{M}\right)$ is model complete, there are an existential $\mathcal{L}$-formula $\psi(\bar{x}, \bar{y})$ and a tuple $\bar{b}$ from $M$ such that $\mathcal{M} \models \delta(\bar{b})$, where

$$
\delta(\bar{y}):=\forall \bar{x}[\varphi(\bar{x}) \leftrightarrow \psi(\bar{x}, \bar{y})]
$$


So $\operatorname{Th}_{\forall \exists}\left(\mathcal{M}_{M}\right) \models \delta(\bar{b})$ by Lemma 13 By compactness, there is an $\forall \exists$-formula $\theta(\bar{y})$ in $\mathcal{L}$ such that $\theta(\bar{b}) \in \operatorname{Th}_{\forall \exists}\left(\mathcal{M}_{M}\right)$ and $\{\theta(\bar{b})\} \models \delta(\bar{b})$. (Without loss of generality, by padding $\delta$, we may assume that any constant symbol appearing in $\theta$ already appears in $\delta$.)

Conversely, suppose that the right-hand side holds. Fix an $\mathcal{L}_{M}$-formula $\varphi(\bar{x}, \bar{a})$, where $\varphi(\bar{x}, \bar{z})$ is an $\mathcal{L}$-formula and $\bar{a}$ is from $M$. Choose $\theta(\bar{y})$ and $\psi(\bar{x}, \bar{z}, \bar{y})$ corresponding to $\varphi(\bar{x}, \bar{z})$. Let $\bar{b}$ be any realization of $\theta(\bar{y})$ in $M$. Then

$$
\mathcal{M} \models \forall \bar{x} \forall \bar{z}[\varphi(\bar{x}, \bar{z}) \leftrightarrow \psi(\bar{x}, \bar{z}, \bar{b})] .
$$

In particular, $\mathcal{M} \models \forall \bar{x}[\varphi(\bar{x}, \bar{a}) \leftrightarrow \psi(\bar{x}, \bar{a}, \bar{b})]$. Thus, every $\mathcal{L}_{M}$-formula is $\operatorname{Th}\left(\mathcal{M}_{M}\right)$ equivalent to an existential $\mathcal{L}_{M}$-formula, $\operatorname{so} \operatorname{Th}\left(\mathcal{M}_{M}\right)$ is model complete.

Corollary 16. If $\mathcal{M}$ and $\mathcal{N}$ are elementarily equivalent $\mathcal{L}$-structures, then the elementary diagram of $\mathcal{M}$ is model complete if and only if the elementary diagram of $\mathcal{N}$ is model complete. In particular, if $T$ is a complete theory and the elementary diagram of some model of $T$ is model complete, then the elementary diagram of every model of $T$ is model complete.

Proposition 17. Let $T$ be any $\mathcal{L}$-theory such that the elementary diagram of some model of $T$ is model complete. Then $T$ is $\exists \forall \exists$-axiomatizable.

Proof. Assume that $\sigma$ is an $\mathcal{L}$-sentence such that $T \models \sigma$. Let $\mathcal{M}$ be a model of $T$ for which the elementary diagram is model complete; so $\operatorname{Th}_{\forall \exists}\left(\mathcal{M}_{M}\right)$ logically implies $\sigma$. Therefore, there is a conjunction $\psi$ of $\forall \exists$-sentences of $\mathcal{L}_{M}$ which logically implies $\sigma$. Since none of these extra constant symbols from $M$ appears in $\sigma$, we can existentially quantify out variables substituted for these constant symbols and obtain a formula of the desired complexity which logically implies $\sigma$.

The following corollary follows immediately from our Main Theorem and Proposition 17

Corollary 18. Every trivial, strongly minimal theory is $\exists \forall \exists$-axiomatizable.

\section{Motivation from and CONSEQuences in COMPUtABle Model THEORY}

The original motivation of our work was a question in computable model theory. One of the goals of computable model theory is to determine the computational complexity of various models of a fixed first-order theory $T$. This is especially important in situations where the models of $T$ are well understood classically, as for uncountably categorical $T$, where, by Baldwin/Lachlan [1], the countable models form an elementary chain of length $\omega+1$ (unless $T$ is totally categorical and thus uninteresting in our context).

We recall some basic definitions of computable model theory.

Definition 19. (1) A countable first-order language $\mathcal{L}$ is computable if its relation, function and constant symbols each form a computable set and the arity of any function or relation symbol of $\mathcal{L}$ can be computed effectively. (Thus, in particular, every finite language is computable.)

For the remainder of the definition, fix a computable language $\mathcal{L}$.

(2) A countable $\mathcal{L}$-model $\mathcal{M}$ is computable (or recursive) if the open (i.e., quantifier-free) diagram of $\mathcal{M}$ forms a computable set of $\mathcal{L}_{M}$-formulas. 
(3) A countable $\mathcal{L}$-model $\mathcal{M}$ is decidable if the (full) elementary diagram of $\mathcal{M}$ forms a computable set of $\mathcal{L}_{M}$-formulas.

Similarly, for any set $X \subseteq \omega$, a countable model is $X$-computable, or $X$-decidable, respectively, if the open diagram, or full diagram, forms an $X$-computable set of $\mathcal{L}_{M}$-formulas.

Of course, the above assumes a Gödel numbering of the symbols of $\mathcal{L}$ and of all $\mathcal{L}_{M}$-formulas, which we suppress here. We also ignore here the question of different numberings of the same model (up to isomorphism), i.e., we call a model $\mathcal{M}$ computable, or decidable, if some isomorphic copy of $\mathcal{M}$ is computable or decidable, respectively, as defined above, or, more precisely, if there is some numbering of $M$ such that the open diagram, or full diagram, respectively, forms a computable set of $\mathcal{L}_{M}$-formulas under the induced numbering of $\mathcal{L}_{M}$-formulas.

Effectivizing the Henkin construction, one can easily see that any decidable firstorder theory $T$ has at least one decidable model $\mathcal{M}$. In the case of an uncountably categorical decidable first-order theory $T$, Harrington [9] and Khisamiev [1] showed that indeed all countable models of $T$ are decidable. If $T$ is uncountably categorical but not decidable, however, it is possible that some of the countable models of $T$ are computable while others are not. This was first shown by Goncharov [5], who constructed an uncountably categorical but not totally categorical theory in infinitely many unary relations for which only the prime model is computable. This naturally leads to the following

Definition 20. Given a computable language $\mathcal{L}$ and an uncountably categorical but not totally categorical $\mathcal{L}$-theory $T$, let $\mathcal{M}_{\alpha}($ for $\alpha \leq \omega)$ be the $\alpha$ th model in the elementary chain of countable models of $T$ given by Baldwin/Lachlan [1]. The spectrum of computable models of $T$ is

$$
\operatorname{SCM}(T)=\left\{\alpha \leq \omega \mid \mathcal{M}_{\alpha} \text { is computable }\right\} .
$$

Clearly (e.g., using algebraically closed fields), $\operatorname{SCM}(T)=\omega \cup\{\omega\}$ is a possible spectrum of computable models. Goncharov's result [5] above can be restated as saying that $\operatorname{SCM}(T)=\{0\}$ is also a possible spectrum of computable models. A number of other possible spectra have been found by Kudaibergenov [13, Khoussainov/Nies/Shore [12] and Nies [16]. The first nontrivial spectrum of computable models (i.e., $\neq \omega \cup\{\omega\}$ ) for a finite language (in fact, for the language of a single binary relation symbol) was found by Herwig/Lempp/Ziegler [10].

In the mid-1990's, Lempp raised the question of how much the various countable models of a fixed uncountably categorical theory $T$ could differ in their computational complexity. Goncharov's theory [5] has a computable prime model while all its countable nonprime models are not only $\mathbf{0}^{\prime}$-computable but can also compute $\mathbf{0}^{\prime}$. Lempp asked if it was possible to construct an uncountably categorical theory $T$ with a computable prime model such that none of the countable nonprime models is even arithmetical. (Here, a model is arithmetical iff it is $X$-computable for some arithmetical set $X$.) For a long time, and with various collaborators, he tried unsuccessfully to code more complicated sets, like $\mathbf{0}^{\prime \prime}$ or $\mathbf{0}^{\prime \prime \prime}$, into the countable nonprime models while keeping the prime model computable.

It turned out the model theory "obstructed" the coding: All the theories for which the spectrum of computable models had been investigated thus far turned out to be strongly minimal and trivial. And for such theories, our Main Theorem answers Lempp's question as follows. 
Theorem 21. Let $\mathcal{M}$ be a computable trivial, strongly minimal model. Then $\mathrm{Th}(\mathcal{M})$ forms a $\mathbf{0}^{\prime \prime}$-computable set of $\mathcal{L}$-sentences, and thus all countable models of $\operatorname{Th}(\mathcal{M})$ are $\mathbf{0}^{\prime \prime}$-decidable (and so, in particular, $\mathbf{0}^{\prime \prime}$-computable).

Proof. By our Main Theorem, $\operatorname{Th}\left(\mathcal{M}_{M}\right)$ is model complete and thus $\forall \exists$-axiomatizable. Then $\operatorname{Th}_{\forall \exists}\left(\mathcal{M}_{M}\right)$ is a $\mathbf{0}^{\prime \prime}$-computable set of formulas which axiomatizes $\operatorname{Th}\left(\mathcal{M}_{M}\right)$, and so $\operatorname{Th}\left(\mathcal{M}_{M}\right)$ and its reduct $\operatorname{Th}(\mathcal{M})$ are $\mathbf{0}^{\prime \prime}$-computable sets of formulas as well. So, by Harrington [9] and Khisamiev 11] relativized to $\mathbf{0}^{\prime \prime}$, any countable model of $\operatorname{Th}(\mathcal{M})$ is $\mathbf{0}^{\prime \prime}$-decidable.

We note that Goncharov and Khoussainov [6, 7] have shown that the hypothesis of strong minimality is necessary in Theorem 21 by exhibiting, for any $n \in \omega$, a non-strongly minimal, trivial, uncountably categorical theory of degree $\mathbf{0}^{(n)}$, all of whose countable models are computable.

We also note here a preliminary result of ours, which preceded the above and which can be seen immediately from Lemma 3

Proposition 22. Let $\mathcal{L}^{\infty}$ be the "infinitary" logic obtained from first-order logic by replacing the usual quantifiers $\exists$ and $\forall$ by the "infinitary" quantifiers $\exists<\infty$ and $\forall^{\infty}$ (i.e., "there exist at most finitely many" and "for cofinitely many"). Then the $\mathcal{L}^{\infty}$-theory (and indeed the elementary $\mathcal{L}^{\infty}$-diagram) of any computable trivial, strongly minimal model is $\mathbf{0}^{\prime}$-computable.

Proposition 17 also allows us to bound the complexity of possible spectra of computable models.

Proposition 23. Let $T$ be an uncountably categorical but not totally categorical $\mathcal{L}$-theory such that the elementary diagram of some model of $T$ is model complete. Then the spectrum of computable models $\operatorname{SCM}(T)$ is a $\Sigma_{5}^{0}$-subset of $\omega \cup\{\omega\}$.

Proof. Suppose $\operatorname{SCM}(T)$ is nonempty since otherwise the result is obvious. Thus $T$ has a computable model $\mathcal{N}$. By Proposition 17, $T$ is $\exists \forall \exists$-axiomatizable and $\mathbf{0}^{\prime \prime}$-computable. Given a computable $\mathcal{L}$-model $\mathcal{M}$, it is thus $\Pi_{4}^{0}$ to check whether $\mathcal{M}=T$; and given any computable $\mathcal{L}$-model $\mathcal{M}=T$ and a tuple $\bar{a} \in M$, it is $\Pi_{3}^{0}$ to check whether $\bar{a}$ is algebraically independent in $\mathcal{M}$, as we will show starting three paragraphs below.

Now let $k$ be the largest size of an algebraically independent subset (over $\emptyset$ ) of the prime model of $T$. Since $T$ is not $\aleph_{0}$-categorical, $k$ is finite. Then a model $\mathcal{M} \models T$ is isomorphic to the model $\mathcal{M}_{\alpha}$ from Definition 20 iff $k+\alpha$ is the largest size of an algebraically independent subset of $\mathcal{M}$. (Here, we are broadly following Nies [16, Proposition 1.1].)

So $n \in \omega$ is in $\operatorname{SCM}(T)$ iff there is an index $e$ for a model $\mathcal{M}$ such that (i) $\mathcal{M} \models T$, (ii) there is an algebraically independent subset of $M$ of size $k+n$, and (iii) all subsets of $M$ of size $k+n+1$ are not algebraically independent. All this constitutes a $\Sigma_{5}^{0}$-condition for $n \in \operatorname{SCM}(T)$. (Whether $\omega \in \operatorname{SCM}(T)$ can be determined nonuniformly.)

We are thus left to show that algebraic independence of a tuple is a $\Pi_{3}^{0}$-property. A tuple $\bar{a}$ from a recursive model $\mathcal{M}$ of $T$ is algebraically independent if and only if for all $\theta, \psi, \bar{b}$ (where $\theta$ is an $\forall \exists$-formula, $\psi$ is existential, and $\bar{b}$ is a tuple from $M)$ and all $a^{*} \in \bar{a}$, one of the following holds:

(a) $(\theta, \psi)$ is not a linked pair (as specified in Definition 14), or

(b) $\mathcal{M} \models \neg \theta(\bar{b})$, or 
(c) $\mathcal{M} \models \neg \psi(\bar{a}, \bar{b})$, or

(d) $\mathcal{M} \models \exists^{\infty} x \psi\left(x, \bar{a}^{\prime}, \bar{b}\right)$ (where $\left.\bar{a}^{\prime}=\bar{a} \backslash\left\{a^{*}\right\}\right)$.

Now (a) and (b) are $\Delta_{3}^{0}$ (since we are already assuming that $\mathcal{M} \models T$ ), (c) is $\Pi_{1}^{0}$, and (d) is $\Pi_{2}^{0}$. In particular, we have universal quantifiers in front of a $\Pi_{3}^{0}$-relation, so algebraic independence is $\Pi_{3}^{0}$.

That the criterion works can be seen as follows. First, suppose that $\bar{a}$ is algebraically independent. Fix any linked pair $(\theta, \psi)$ and $\bar{b}$ such that $\mathcal{M} \models \theta(\bar{b}) \wedge \psi(\bar{a}, \bar{b})$. Then $\mathcal{M}=\eta(\bar{a})$, where $\eta(\bar{x}):=\exists \bar{y}[\theta(\bar{y}) \wedge \psi(\bar{x}, \bar{y})]$. Since $\bar{a}$ is algebraically independent, for any $a^{*} \in \bar{a}$ there are infinitely many $c$ in $M$ such that $\mathcal{M} \models \eta\left(c \bar{a}^{\prime}\right.$ ) (where $\left.\bar{a}^{\prime}=\bar{a} \backslash\left\{a^{*}\right\}\right)$. For each such $c$, choose $\bar{b}^{\prime}$ such that $\theta\left(\bar{b}^{\prime}\right) \wedge \psi\left(c \bar{a}^{\prime}, \bar{b}^{\prime}\right)$ holds in $\mathcal{M}$. Since $(\theta, \psi)$ is a linked pair, this implies that $\psi\left(c \bar{a}^{\prime}, \bar{b}\right)$ holds in $\mathcal{M}$. Thus (d) holds.

Conversely, suppose that $\bar{a}$ is not algebraically independent. Then some $a^{*} \in \bar{a}$ depends on $\bar{a}^{\prime}=\bar{a} \backslash\left\{a^{*}\right\}$. So, by the above argument, there is an $\mathcal{L}$-formula $\varphi(\bar{x})$ that "witnesses it", i.e., $\mathcal{M} \models \varphi(\bar{a})$ but there are only finitely many $c$ such that $\mathcal{M}=\varphi\left(c \bar{a}^{\prime}\right)$. Now choose a linked pair $(\theta, \psi)$ for $\varphi$ using Proposition 15 and choose any $\bar{b}$ from $M$ such that $\theta(\bar{b})$ holds. It is now easy to check that conditions (a)-(d) all fail.

Corollary 24. For any strongly minimal, trivial, not totally categorical theory $T$, the spectrum of computable models $\operatorname{SCM}(T)$ is a $\Sigma_{5}^{0}$-subset of $\omega \cup\{\omega\}$.

Proof. Immediate by the Main Theorem and Proposition 23. (Note that triviality implies that $k \leq 1$ in the proof of Proposition 23],

\section{REFERENCES}

1. Baldwin, John T. and Lachlan, Alistair H., On strongly minimal sets, J. Symbolic Logic 36 (1971), 79-96. MR 44:3851

2. Buechler, Steven A., Essential Stability Theory, Springer-Verlag, Heidelberg, 1996. MR 98j:03050

3. Chang, C. C. and Keisler, H. Jerome, Model Theory (3rd edition), North-Holland, Amsterdam, 1990. MR 91c:03026

4. Ershov, Yuri L. and Goncharov, Sergei S. Constructive models, Siberian School of Algebra and Logic, Consultants Bureau, New York, 2000. MR 2002a:03069

5. Goncharov, Sergei S., Constructive models of $\aleph_{1}$-categorical theories, Mat. Zametki 23 (1978), 885-888. MR 80g:03029

6. Goncharov, Sergei S. and Khoussainov, Bakhadyr M. On the complexity of theories of computable $\aleph_{1}$-categorical models, Vestnik NGU, Ser. Mat.-Mekh.-Inform. 1 (2) (2001), 63-76.

7. Goncharov, Sergei S. and Khoussainov, Bakhadyr M. Complexity of theories of computable models, Algebra and Logic, to appear.

8. Harizanov, Valentina S., Pure computable model theory, in: "Handbook of recursive mathematics", Vol. 1, North-Holland, Amsterdam, 1998, pp. 3-114. MR 2000f:03108

9. Harrington, Leo, Recursively presented prime models, J. Symbolic Logic 39 (1974), 305-309. MR 50:4292

10. Herwig, Bernhard; Lempp, Steffen; and Ziegler, Martin, Constructive models of uncountably categorical theories, Proc. Amer. Math. Soc. 127 (1999), 3711-3719. MR 2000b:03129

11. Khisamiev, Nazif G., On strongly constructive models of decidable theories, Izv. Akad. Nauk Kazakh. SSR Ser. Fiz.-Mat. 35 (1) (1974), 83-84. MR 50:6824

12. Khoussainov, Bakhadyr M.; Nies, André O.; and Shore, Richard A., Computable models of theories with few models, Notre Dame J. Formal Logic 38 (1997), 165-178. MR 99c:03049

13. Kudaibergenov, Kanat Zh., Constructivizable models of undecidable theories, Sibirsk. Mat. Zh. 21 (5) (1980), 155-158, 192. MR 82h:03040

14. Kueker, David W., Weak invariance and model completeness relative to parameters, in preparation. 
15. Marker, David, Non $\Sigma_{n}$ axiomatizable almost strongly minimal theories, J. Symbolic Logic 54 (1989), 921-927. MR 90g:03037

16. Nies, André O., A new spectrum of recursive models, Notre Dame J. Formal Logic 40 (1999), 307-314. MR 2002e:03066

17. Pillay, Anand, An introduction to stability theory, Oxford University Press, New York, 1983. MR 85i:03104

18. Shelah, S. Classification theory and the number of nonisomorphic models, 2nd edition, NorthHolland Publishing Co., Amsterdam, 1990. MR 91k:03085

Institute of Mathematics, Siberian Branch of the RAS, 630090 Novosibirsk, Russia

E-mail address: gonchar@math.nsc.ru

Department of Mathematics, George Washington University, Washington, DC 20052

E-mail address: harizanv@gwu.edu

Department of Mathematics, University of Maryland, College Park, Maryland 20742

E-mail address: mcl@math.umd.edu

Department of Mathematics, University of Wisconsin, Madison, Wisconsin 53706

E-mail address: lempp@math.wisc.edu

Department of Mathematics, University of Wisconsin, Madison, Wisconsin 53706

E-mail address: mccoy@math.wisc.edu

Current address: P.O. Box 668, Notre Dame, Indiana 46556 